\title{
Investigation into the controversial association of Streptococcus gallolyticus with colorectal cancer and adenoma
}

\author{
Ahmed S Abdulamir*1,3, Rand R Hafidh ${ }^{4}$, Layla K Mahdi², Tarik Al-jeboori ${ }^{3}$ \\ and Fatimah Abubaker 4
}

\begin{abstract}
Address: ${ }^{1}$ Microbiology Research Department, Faculty of Medicine, University Putra Malaysia, 43400, UPM, Serdang, Selangor Darul Ehsan, Malaysia, ${ }^{2}$ School of Molecular and Biomedical Science, University of Adelaide, South Australia, 5005, ${ }^{3}$ Medical Microbiology Department, College of Medicine, Alnahrain University, 14222, Iraq and "4nstitute of bioscience, University Putra Malaysia, 43400, UPM, Serdang, Selangor
\end{abstract} Darul Ehsan, Malaysia

Email: Ahmed S Abdulamir* - ahmsah73@yahoo.com; Rand R Hafidh - ranria77@yahoo.com; Layla K Mahdi - laylakhm@yahoo.com; Tarik Aljeboori - tarik_aljeboori@yahoo.com; Fatimah Abubaker - fatim@putra.upm.edu.my

* Corresponding author

Published: 19 November 2009

BMC Cancer 2009, 9:403 doi:10.1 |86/147|-2407-9-403
Received: 12 December 2008

Accepted: 19 November 2009

This article is available from: http://www.biomedcentral.com/I47I-2407/9/403

(C) 2009 Abdulamir et al; licensee BioMed Central Ltd.

This is an Open Access article distributed under the terms of the Creative Commons Attribution License (http://creativecommons.org/licenses/by/2.0), which permits unrestricted use, distribution, and reproduction in any medium, provided the original work is properly cited.

\begin{abstract}
Background: The seroprevalence of IgG antibodies of Streptococcus gallolyticus subspecies gallolyticus, CIP 105428, was evaluated to investigate the controversial association of S. gallolyticus with colorectal carcinoma and adenoma in attempt to investigate the nature of such association if any, by exploring the mRNA expression of NF-KB and IL-8. Moreover, the serological behavior of S. gallolyticus IgG antibodies was compared to that of an indicator bacterium of bowel, Bacteroides fragilis.

Methods: ELISA was used to measure IgG antibodies of $S$. gallolyticus and B. fragilis in sera of 50 colorectal cancer, 14 colorectal adenoma patients, 30 age- and sex- matched apparently healthy volunteers $(\mathrm{HV})$ and 30 age- and sex- matched colonoscopically-proven tumor-free control subjects. NF- $\mathrm{kB}$ and IL-8 mRNA expression was evaluated in tumorous and non-tumorous tissue sections of carcinoma and adenoma patients in comparison with that of control subjects by using in situ hybridization assay.

Results: Colorectal cancer and adenoma patients were associated with higher levels of serum S. gallolyticus IgG antibodies in comparison with HV and control subjects $(P<0.05)$ while no similar association was found with serum $\lg G$ antibodies of $B$. fragilis $(P>0.05)$. ELISA cutoff value for the seropositivity of $S$. gallolyticus $\lg G$ was calculated from tumor-free control group. The expression of NF- $\kappa B$ mRNA was higher in tumorous than non-tumorous tissue sections of adenoma and carcinoma, higher in carcinoma/adenoma sections than in control subjects, higher in tumorous sections of carcinoma than in adenoma patients, and higher in $S$. gallolyticus $\lg G$ seropositive than in seronegative groups in both tumorous and non-tumorous sections $(P<0.05)$. IL-8 mRNA expression in tumorous sections of adenoma and carcinoma was higher than in non-tumorous sections, higher in carcinoma/adenoma than in control subjects, and higher in $S$. gallolyticus IgG seropositive than in seronegative groups in tumorous rather than non-tumorous sections $(P<0.05)$.
\end{abstract}

Conclusion: S. gallolyticus most likely plays an essential role in the oncogenic progression of normal colorectal mucosa to adenoma and to CRC. This promoting/propagating role of $\mathrm{S}$. gallolyticus might take place by utilizing certain inflammatory, anti-apoptotic, and angiogenic factors of transformation including NF-KB and IL-8. 


\section{Background}

Colorectal cancer (CRC) is the fourth commonest form of cancer occurring worldwide. The number of new cases of colorectal cancer has been increasing rapidly since 1975 [1]. Several studies have associated bacterial infections to carcinogenesis [2,3]. CRC was associated with Streptococcus bovis (S. bovis); the incidence of the association of colonic neoplasia with $S$. bovis has been determined as $18 \%$ to $62 \%[4,5]$. Colonic neoplasia may arise years after the presentation of the condition of bacteremia or infectious endocarditis of $S$. bovis $[5,6]$.

Prior to the description of $S$. gallolyticus, it was reported that among $S$. bovis biotypes identified by the API Rapid Strep system and cellular fatty acid content, biotype I was more likely than biotype II to be associated with both endocarditis and malignant or premalignant colonic lesion [7]. Following the description of S. gallolyticus, Devriese team showed that the bacterial isolates, which were studied previously and derived from patients with endocarditis and associated with colonic cancers and identified by conventional techniques as $S$. bovis, were in fact S. gallolyticus [8]. They suggested that S. gallolyticus is more likely to be involved in human infections than $S$. bovis and most of $S$. gallolyticus strains belong to the socalled $S$. bovis biotype I and a few belong to $S$. bovis biotype II/2. Recently S. gallolyticus subspecies gallolyticus has become the most implicated agent in the association with CRC as Schlegel et al. stated that most of the human strains isolated from blood or feces were Streptococcus gallolyticus which is often responsible for endocarditis cases associated with a colonic cancer [9].

After the new species, S. gallolyticus, was assigned, there has been no specific serological study done for the association between S. gallolyticus and CRC or colorectal adenoma. Therefore, we conducted a serological investigation of S. gallolyticus IgG antibodies in CRC and colorectal adenoma patients in comparison with normal individuals. To keep the scientific fidelity, we accompanied another intestinal bacterium, namely Bacteroides fragilis (B. fragilis), strain ATCC 25285. B. fragilis is one of the most dominant bacteria in the normal flora of humans' large intestine and present in bowel at incidence of $100 \%$ [10]. B. fragilis was selected for this comparison because $B$. fragilis is confined to the bowel and isolated from the blood circulation by an integral mucosal barrier; any breach, say degenerative lesion or ulceration, in the mucosal barrier of the bowel leads to showering of huge amount of $B$. fragilis into blood circulation which results in a vigorous immune response [11]. Although no quantitative comparison was aimed between the seroprevalence of $B$. fragilis and S. gallolyticus, as they are of different species, we intended to compare the behavior or trend of the seroprevalence of $B$. fragilis lipopolysaccharides (LPS) IgG antibodies among
CRC, adenoma and normal subjects to that of our target bacteria, S. gallolyticus cell wall antigens IgG antibodies. The reason behind that was to assess whether the association of S. gallolyticus with CRC and adenoma is specific or other bowel bacteria are associated too through a nonspecific entry of bowel bacteria into blood circulation via tumor lesion. In addition, LPS of $B$. fragilis is a specific antigen and not shared with other Gram negative/positive bacteria. It was reported that no cross-reactivity between $B$. fragilis LPS and LPS of other bacteria was observed and $B$. fragilis LPS contains an immunodominant antigenic determinant common to almost all $B$. fragilis isolates $[11,12]$. Moreover, it was revealed that anti- $B$. fragilis IgM, IgG, and IgA antibodies in mice and human serum were measured reliably by using $B$. fragilis LPS in enzyme immune assays $[13,14]$.

Previous experiments by our team showed no link between S. gallolyticus-associated CRC and a number of tumor suppressor proteins, p53, p21, and p27 [unpublished data] as these factors were found to be greatly associated with the transformation process of colorectal mucosa to adenoma and then to carcinoma [15]. Therefore, in the current study, un-researched before, target molecules, namely, nuclear factor kappa-B (NF-кB) and interleukin-8 (IL-8) were studied in relation with $S$. gallolyticus as well as the transformation of colorectal mucosa to adenoma and carcinoma. Accordingly, the mRNA expression of NF- $\kappa \mathrm{B}$, a central transcriptional factor for inflammation [16] and IL-8, a powerful angiogenic chemokine which is frequently related to bacterial carcinogenesis e.g. H. pylori [17], were evaluated using in situ hybridization (ISH) assay.

\section{Methods \\ The population of the study}

CRC patients were those who attended several Gastroenterology and Hepatology Centers in the state of Selangor, Malaysia from March 2006 to December 2007, that underwent elective surgical resection of colorectal cancer. Adenoma patients were those who were referred to do colonoscopy due to various reasons and resection of intestinal polyps was done. Twenty seven men and 23 women with primary colorectal adenocarcinoma were included in this study prior to any chemotherapy. On the other hand, seven men and seven women with colorectal adenoma who had already undergone colonoscopical resection of adenomatous polyps were involved in this study. The medical history of the involved patients in this study was evaluated; no one had history of gastrointestinal disease or ulceration which might affect the seroprevalence of $S$. gallolyticus or B. fragilis. On the other hand, 30 age- and sex- matched control subjects were involved. They were referred to hospitals for doing colonoscopy for various reasons in whom normal colonic mucosa was confirmed 
and no other gastrointestinal disease or history of gastrointestinal diseases and ulcerations were found. Colonoscopical biopsy was taken from control subjects to compare the level of IL- 8 and NFKB mRNA expression with that in mucosal colonic tissues from patients with adenoma and CRC. Moreover, blood samples were taken from the control group to calculate the seropositivity cutoff value for $S$. gallolyticus IgG antibodies. The resultant cutoff value was applied on CRC and adenoma patients as well as on age- and sex- matched 30 apparently healthy volunteers group (HV) who agreed to do colonoscopy in case results of ELISA would show high level of $S$. gallolyticus IgG antibodies. HV were medically examined and their medical records and history were reviewed and no major or gastrointestinal illness was found. Written consents were officially obtained from all participants in this study. The study was carried out in the scope of Helsinki declaration of ethical principles of medical research and permission was granted from the Ethics Committee of biomedical research of University Putra Malaysia.

\section{Sampling and processing of specimens}

Samples of 3 to $5 \mathrm{ml}$ of blood for serum isolation were withdrawn from control and adenoma groups at time of colonoscopy, from HV group after taking the medical history, and from CRC patients 2-3 days before surgery. Regarding the histopathology, a set of steps was pursued under the supervision of a pathologist to minimize as could as possible the fixation-related loss of mRNA. These steps were minimal prefixation time of 1 hour, the use of cold $4 \%$ paraformaldehyde, cold fixation at $4{ }^{\circ} \mathrm{C}$, and short duration of fixation, up to 5 hours [18]. It was stated that no significant loss of nucleic acids was observed within the first 3 days of fixation-paraffin embedding [19]. Therefore, paraffin-embedded sections were processed for ISH examination in a period of not more than 3 days. Moreover, the scoring system used in this study did not rely on the quantitative measurement of staining intensity. Therefore, mRNA loss, which affects mainly the intensity of staining, was believed to affect the results of this study minimally. To evaluate the stringent conditions pursued for minimizing mRNA loss, the ISH immunostaining was compared between 6 randomly selected paraffin embedded sections and cryostat sections. Despite the bit lower intensity of staining, the mean percentage of the positively stained cells was not changed. Hence it was confirmed that no loss of mRNA took place which could affect ISH results. Each histopathological paraffin block of excisional biopsies of CRC patients and punch biopsies of control subjects and adenoma patients were sectioned into $4 \mathrm{um}$ thick sections. Histopathological sections were made from both tumorous and non-tumorous tissues for each CRC (resection safe margins) and adenoma patient (punch biopsy 3-4 cm away from the polyp). Hematoxylin and Eosin slides were prepared and examined by a his- topathologist for confirming the histopathological diagnosis, the grade of $\mathrm{CRC}$, and the type and degree of dysplasia of adenoma tissue sections.

\section{Extraction of cell wall antigens of S. gallolyticus and LPS of B. fragilis}

The reference strain, $S$. gallolyticus subspecies gallolyticus CIP 105428 (Insitut de Louis Pasteur, France) was used. The extraction of cell wall antigens of S. gallolyticus was conducted by using the lysozyme method. Sufficient amount of the cultured reference bacteria in Columbia agar (Oxoid, UK) with 5\% horse blood was obtained by making bacterial suspension in $30 \mathrm{mM}$ (pH 8) Tris buffer (Fluka, Switzerland). The suspension was centrifuged at $3500 \mathrm{~g}$ for 10 minutes at $4^{\circ} \mathrm{C}$. Pellet was washed 3 times in $30 \mathrm{mM}$ Tris buffer ( $\mathrm{pH} \mathrm{8)}$ by centrifugation at $3500 \mathrm{~g}$ for 5 minutes at $4{ }^{\circ} \mathrm{C}$. Pellet of washed bacteria was resuspended in a solution containing $4.75 \mathrm{ml}$ of $30 \mathrm{mM}$ Tris buffer (pH 8), 3 mM magnesium chloride (Sigma, USA), $25 \%$ sucrose (Fluka, Switzerland) and $0.25 \mathrm{ml}$ of lysozyme $(0.6 \mathrm{mg} / \mathrm{ml})$ (Sigma, USA). The lysate solution was incubated for 2 hours at $37^{\circ} \mathrm{C}$ and was then centrifuged at $3000 \mathrm{~g}$ for 5 minutes at $4^{\circ} \mathrm{C}$. Then, the supernatant was collected, which became the solution of cell wall antigens of the reference strain, S. gallolyticus [20]. The concentration of cell wall antigens was measured by Biurette method.

The extraction of $B$. fragilis LPS was obtained by the phenol-water extraction method, followed by the phenolchloroform-petroleum ether extraction, as described by [21].

\section{ELISA}

For S. gallolyticus, 96-wells microtiter plate (Sterilin, UK) was coated with $40 \mu \mathrm{g} / \mathrm{ml}$ of cell wall antigen while, for $B$. fragilis, the microtiter plate was coated with $10 \mu \mathrm{g} / \mathrm{ml}$ of LPS extract. The concentration of coating antigens for the studied bacteria was determined after a series of standardization steps. The microtiter plates were incubated in a humid chamber for 2 hours at $37^{\circ} \mathrm{C}$ and were then washed and stored at $-20^{\circ} \mathrm{C}$ until further use. It is noteworthy to mention that the cell wall antigens of $S$. gallolyticus had already been treated with $100 \mu \mathrm{l}$ a well of 0.01 $M$ sodium periodate (Sigma, USA) in PBS for two hours at room temperature to destroy the common polysaccharides antigen of group D. After 3 times washing, $50 \mu \mathrm{l}$ sera of CRC (50), adenoma (14), control subjects (30), and healthy volunteers (30) were pipetted into microtiter plates and incubated for 2 hours at room temperature. For each run, two wells were dispensed with $50 \mu$ l of diluting buffer as a negative control and two wells were dispensed with known positive sera for either S. gallolyticus or B. fragilis. After 3 rounds of washing, $50 \mu \mathrm{l} /$ well of 1:40,000 diluted horseradish peroxidase anti-human IgG conju- 
gates (Sigma, USA) were dispensed and incubated for 2 hours at room temperature. Then, $50 \mu \mathrm{l} /$ well of chromogen-substrate OPD.2HCL (Abbott, USA) were pipetted into wells and incubated in dark for 15 minutes at room temperature. Optical density was read by ELISA reader (Organic Technica, Spain) at $492 \mathrm{~nm}$ [22].

\section{Calculation of the cutoff value for S. gallolyticus seropositivity}

The cut off value is considered as the upper limit above which all of readings are considered positive. ELISA readings of control subjects $(n=30)$ were used to calculate the cutoff value according to the following formula [23]:

$99 \%$ Confidence interval (cutoff value) $=$ mean $+2.462 \times$ Standard error $_{\text {mean }}$ [23] (2.462): taken from the table of student's $t$-test under the $P=0.01$ for the 29 degrees of freedom.

The cutoff value was used to demarcate between the S. gallolyticus- seropositive and seronegative subjects in the participants of this study other than control group, namely CRC, adenoma, and HV groups

\section{In situ hybridization assay}

For each run of ISH, one negative control tissue section (diluting buffer instead of probes), one positive tissue section (already tested as strongly positive), and one endogenous positive probe control were used. Biotinylated long DNA probe for human NF- $\kappa$ B mRNA and human IL-8 mRNA were used (Maximbio, USA). The used procedure was according to "DNA probe Hybridization/Detection System - in situ Kit" (Maximbio, USA).

Slides were baked overnight at $70^{\circ} \mathrm{C}$ and deparaffinized in xylene (Merck, Germany) and descending grades of ethanol (Merck, Germany) starting from 100\%. Freshly diluted $1 \times$ proteinase $\mathrm{K}$ (Sigma, USA) solution was applied for $15 \mathrm{~min}$ at $37^{\circ} \mathrm{C}$. The working solutions used for probes were $10 \% \mathrm{v} / \mathrm{v}$ for NF- $\kappa \mathrm{B}$ and $7 \% \mathrm{v} / \mathrm{v}$ for IL-8 mRNA. Ten $\mu$ l of the working cDNA probe was added onto each slide. Slides were incubated in a humid chamber overnight at $37^{\circ} \mathrm{C}$. Next day after washing slides, RNase A (Maximbio, USA) was added for $30 \mathrm{~min}$ to abolish any unbound RNA. Slides were then washed three times with a pre-warmed protein block for 3 minutes at $37^{\circ} \mathrm{C}$. One to two drops of alkaline phosphatase-streptavidin conjugate (Abbott, USA) were added onto tissue sections for 1.5 hour at $37^{\circ} \mathrm{C}$. Then, 1-2 drops of NBT/BCIP (Abbott, USA) substrate were placed on tissue sections at $37^{\circ} \mathrm{C}$ until color was developed. Dark blue colored precipitate was seen in positive cells. Slides were counterstained by nuclear fast red, dehydrated by graded alcohols, and mounted with a permanent-mounting DPX medium.

\section{Staining analysis}

The scoring systems for in situ hybridization staining of NF- $\kappa \mathrm{B}$ and IL-8 mRNA calculate the percentage of the glandular mucosal (adeno) cells, which were stained with nuclear blue/black color, out of total cells in 5 high power fields. The scores for NF- $\kappa \mathrm{B}$ mRNA staining are; negative for less than $5 \%$ staining, low for $5-25 \%$ staining, intermediate for $26-50 \%$ staining, and positive for more than $50 \%$ staining [24]. The scores for IL- $8 \mathrm{mRNA}$ are; score 1 for $1-10 \%$ staining, score 2 for $11-50 \%$ staining, and score 3 for $51-100 \%$ staining [25]. In addition, stromal cells rather than glandular mucosal cells were observed too. The percentage of IL-8- or NF- $\kappa \mathrm{B}$ - positively stained stromal cells were calculated out of total cells in 5 high power fields. However, no specific scoring system was found for stromal cells of human colonic tissues. Therefore, the ISH staining percentage of stromal cells was used for comparisons without using a predetermined scoring system.

\section{Statistical analysis}

SPSS software version 12 for Windows (SPSS Inc., USA) and Excel XP (Microsoft, USA) were used. To validate the scoring systems used in this study, two sets of statistical analyses were used. The first set, Chi square for independence and Mann-Whitney tests were used for the scoring systems of NF- $\mathrm{KB}$ and IL- 8 respectively. The second set, a univariate student $t$ test was used for direct comparisons of the mean percentages of the positively stained cells. On the other hand for stromal cells, only student $t$ test for mean percentages of the positively stained cells was used. Pearson's correlation coefficient was used to correlate the expression of IL- 8 mRNA and NF- $\kappa$ B mRNA with $S$. gallolyticus IgG antibodies in both CRC and adenoma patients. $P$ values less than 0.05 were considered significant.

\section{Results \\ Demographic and histopathological features of CRC and adenoma patients}

The mean age of CRC and adenoma patients was 57.08 years, ranged between 43 and 76 years, and 50.1 years, ranged between 37 and 70 years, respectively. On the other hand, the mean age of control and HV groups was 53.5 years, ranged between 38 and 78 years, and 52.8 years, ranged between 41 and 73 years, respectively. The whole of CRC cases were of adenocarcinoma; 32 cases were left-sided, 12 right-sided, and 6 at transverse colon. Seven out of 32 left-sided CRC were located in the rectum. Three (6\%) of CRC patients were presented at B1, $5(10 \%)$ at B2, $5(10 \%)$ at C1, $7(14 \%)$ at C2, and $30(60 \%)$ at D stages. It was found that $30(60 \%)$ of histological sections of CRC patients were poorly differentiated and 20 (40\%) were mild-moderate differentiated. For adenoma, it was found that 6 patients $(43 \%)$ were of villous type, 5 (35.7\%) were of tubulovillous type, and only $3(21.4 \%)$ were of tubular adenomatous polyps. 
Seroprevalence of IgG antibodies for S. gallolyticus and B. fragilis

It was found that both CRC and adenoma patients showed significantly higher $S$. gallolyticus IgG antibodies than control group. Mean ELISA readings in terms of optical density (OD) were used to measure the serum level of S. gallolyticus IgG antibodies in both CRC (0.158 \pm 0.032$)$, and adenoma patients $(0.173 \pm 0.024)$ which were higher than that of HV group $(0.064 \pm 0.011)$ and control subjects $(0.046 \pm 0.024)(P<0.05)$. However, there was no significant difference between the serum level of $S$. gallolyticus IgG antibodies of CRC and of adenoma patients ( $P$ $>0.05$ ) or between the serum level of $S$. gallolyticus IgG antibodies of HV group and of control group ( $\mathrm{P}>0.05$ ) (figure 1). On the other hand, it was found that the seroprevalence of $B$. fragilis, as an indicator for any access of the large intestine flora into the circulation, was not higher in CRC patients $(0.166 \pm 0.013)$ and colorectal adenoma patients $(0.178 \pm 0.032)$ than in HV members $(0.176 \pm 0.014)$ and control group $(0.18 \pm 0.02)(\mathrm{P}>$ 0.05 ) (figure 1 ). In addition, there was no significant difference found in the serum level of $S$. gallolyticus IgG antibodies among CRC patients in terms of age, sex, and tumor site, colon versus rectum, and colon segments $(\mathrm{P}>$ 0.05).

The cutoff value for $S$. gallolyticus seroprevalence, which was calculated from the control group, was equal to OD value of 0.07 . By applying this cutoff value on CRC, adenoma, and HV groups, it was found that the percentage of seropositive subjects for $S$. gallolyticus IgG antibodies in
CRC group, 34 out of 50 (68\%), was close to that of adenoma group, 11 out of 14 (78\%) ( $\mathrm{P}>0.05)$. However, these percentages were remarkably higher than that of $\mathrm{HV}$ group, 5 out of $30(16.66 \%)(P<0.05)$ (figure 2$)$. Accordingly, the use of this cutoff value in ELISA for the seropositivity of S. gallolyticus IgG antibodies yielded sensitivity of $68 \%$ for CRC detection and $78 \%$ for adenoma detection and specificity of $83.33 \%$. There was no significant difference in age and sex between seropositive and seronegative groups of CRC, adenoma, and HV groups $(\mathrm{P}>0.05)$. The 5 seropositive members of HV group were traced and 4 of them volunteered to do colonoscopy. Three members showed normal colonic mucosa and one showed begnine hyperplastic polyp $(0.7 \mathrm{~cm}$ in diameter $)$ in the distal colon. There was no particular history of CRC and colorectal adenoma in the seropositive members of HV group.

\section{In situ hybridization expression of NF- $K B$ mRNA and IL-8 mRNA in CRC and adenoma patients}

ISH immunostaining of mucosal (adeno) cells

The expression level of NF- $\kappa \mathrm{B}$ mRNA and IL- 8 mRNA (figure 3) was tested by ISH staining in tumorous versus nontumorous tissue sections in CRC and adenoma patients (Table 1 and 2) and normal mucosal tissue sections from control subjects versus tumorous and non-tumorous tissue sections of CRC and adenoma patients (Table 1 and 2). In addition, the mRNA expression of NF- $\kappa B$ and IL- 8 was tested in tumorous and non-tumorous tissue sections of CRC versus that of adenoma patients (Tables 3 and 4) and in CRC S. gallolyticus seropositives (CRC-Sg+ve) ver-
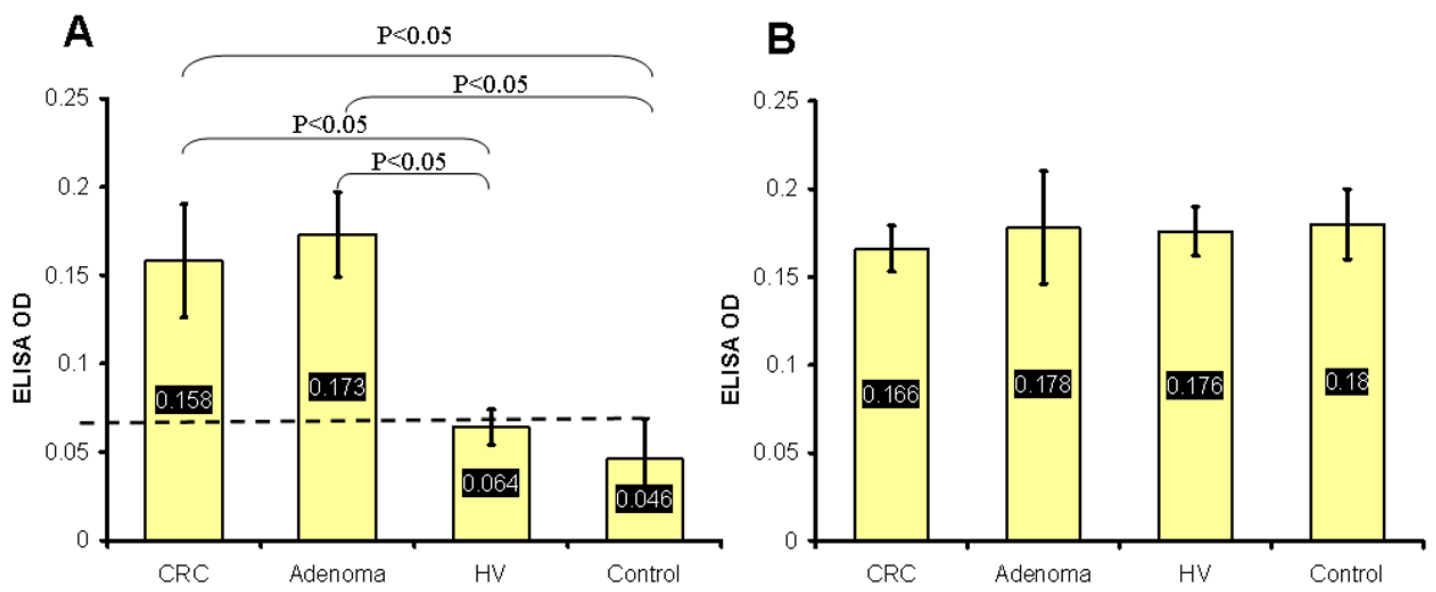

\section{Figure I}

Serum levels of IgG antibodies. (A) The serum level of IgG antibodies of S. gallolyticus in terms of ELISA OD values. The high level of serum IgG antibodies of $S$. gallolyticus was detected in both CRC and adenoma patients which were significantly higher than that of HV and control group. The dashed line represents the ELISA cutoff value (OD 0.07) for the S. gallolyticus seropositivity measured from the tumor-free control group and applied on CRC, adenoma and HV groups. (B) The serum level of IgG antibodies of $B$. fragilis, an indicator bacterium for comparison, was not different among CRC, adenoma, $\mathrm{HV}$, and control groups. 


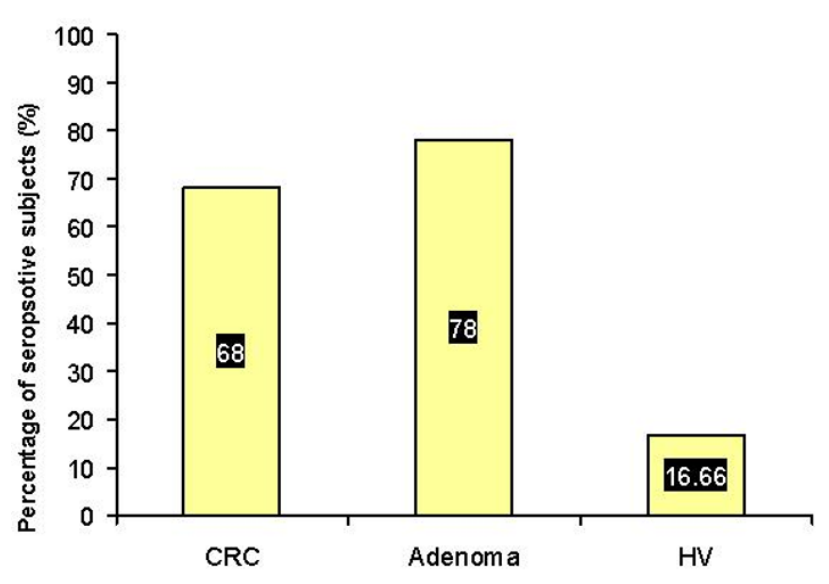

Figure 2

The percentage of seropositives for S. gallolyticus IgG antibodies in CRC, adenoma, and HV groups depending on the cutoff value calculated from the tumorfree control group. Colorectal adenoma and CRC groups showed high percentage of S. gallolyticus IgG seropositives while HV group showed much lower percentage.

sus that of CRC S. gallolyticus seronegatives (CRC-Sg-ve) (Tables 3 and 4 ).

At first, it was important to explore whether the mRNA expression of NF-KB and IL-8 is intensified in tumor cells of CRC and adenoma in comparison with normal mucosal tissues of control subjects and the surrounding mucosal tissue (non-tumorous) or not. It was found that the mRNA expression of NF- $\mathrm{KB}$ and IL-8 in the tumorous tissue sections of CRC and adenoma patients was higher than in the normal mucosal tissue sections of the control subjects and that of the corresponding non-tumorous tissue sections $(\mathrm{P}<0.05)$ (Table 1 and 2$)$. This indicated a significant association of the expression of NF- $\kappa \mathrm{B}$ mRNA and IL-8 mRNA with the tumorous tissues of both CRC and adenoma patients rather than the surrounding nontumorous tissues or normal mucosal tissues. Moreover, the non-tumorous tissue sections of CRC patients but not adenoma patients showed higher levels of expression of NF- $\kappa B$ mRNA but not IL-8 mRNA than that of normal mucosal tissues of control subjects (Table 1). This provided evidence that NF-kB mRNA expression, unlike IL-8, increases in the tumor-adjacent normal mucosal cells in patients with CRC but not adenoma.

The NF- $\kappa \mathrm{B}$ mRNA expression in tumorous tissue sections of CRC patients was associated with the seropositivity of $S$. gallolyticus IgG antibodies in that its expression was higher in CRC-Sg+ve group than in CRC-Sg-ve group ( $\mathrm{P}<$ $0.05)$. And NF- $\mathrm{B}$ mRNA expression was higher in carcinoma than in adenoma patients $(\mathrm{P}<0.05)$ (Table 3$)$.
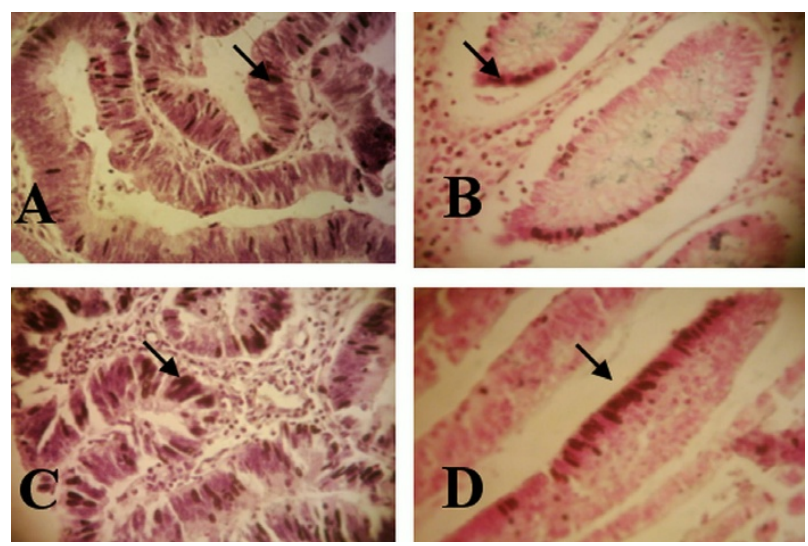

Figure 3

Examples for NF- $\kappa$ B mRNA expression in the tumorous sections of CRC patients. (A) positive tissue section, $>50 \%$ stained cells, and (B) tissue section, $5 \%-25 \%$ stained cells with stained stromal cells. Examples for IL-8 mRNA expression in CRC patients at (C) score 2, $11 \%-50 \%$ stained cells and at (D) score I, I\%-I0\% stained cells. Cells stained positive are shown with dark color of NBT/BCIP in the nuclei of glandular cells (adenocarcinoma cells) $(\times 400)$ and pointed by black bold arrow.

However, it was found that, just like the tumorous tissue sections, the expression of NF- $\mathrm{kB}$ mRNA in non-tumorous tissue sections was higher in CRC-Sg+ve group than in CRC-Sg-ve group $(\mathrm{P}<0.05)$ but was not higher in CRC than in adenoma $(P>0.05)$ (Table 3$)$. This indicated that high levels of NF- $\mathrm{kB}$ mRNA expression were associated significantly with the seropositivity of S. gallolyticus IgG antibodies in both tumorous cells and histologically normal adjacent cells making NF- $\mathrm{KB}$ as a remarkable factor associated with the hypothesis of S. gallolyticus-associated/ triggered CRC. Moreover, NF- $\mathrm{kB}$ was shown to be associated with the transformation process from adenoma to CRC. On the other hand, the level of NF-kB mRNA expression in CRC patients was not related with staging or with tumor grade $(\mathrm{P}>0.05)$. It is noteworthy to mention that the positive expression of NF- $\mathrm{KB}$ was $0 \%$ in negative control tissue section, and 73\% in positive control tissue section.

For IL-8 mRNA expression, the negative control sections showed score 0 and the positive control section showed score 2. In tumorous tissue sections, IL-8 mRNA expression was associated with the seropositivity of $S$. gallolyticus IgG antibodies in that its expression was higher in CRC$\mathrm{Sg}+\mathrm{ve}$ group than in CRC-Sg-ve group $(\mathrm{P}<0.05)$. On the other hand, there was no significant difference between IL-8 mRNA expression in CRC versus adenoma patients ( $P$ $>0.05$ ) (Table 4). In the non-tumorous tissue sections, it was found that the positive expression of IL-8 mRNA was not higher in CRC-Sg+ve group than in CRC-Sg-ve group 
Table I: The expression of NF- $K B$ mRNA in the tumorous and the non-tumorous tissue sections of CRC and adenoma patients as well as in normal tissue sections of control subjects.

\begin{tabular}{|c|c|c|c|c|}
\hline Tissue sections of NF- $\kappa B$ & $\begin{array}{c}\text { Positive NF- } \kappa \text { B mRNA } \\
\text { expression N (\%) }\end{array}$ & $\begin{array}{l}\chi^{2} \text { test } \\
P \text { value }\end{array}$ & $\begin{array}{c}\text { Mean \% of NFKB-positively } \\
\text { stained cells }\end{array}$ & $\begin{array}{c}\text { P value for positively stained } \\
\text { cells }\end{array}$ \\
\hline \multicolumn{5}{|l|}{ CRC: } \\
\hline Tumorous & $36 / 50(72)$ & 0.0006 & $74.7 \pm 4.7$ & $<0.01$ \\
\hline Non-tumorous & $19 / 50(38)$ & & $36.23 \pm 3.6$ & \\
\hline Tumorous & $36 / 50(72)$ & $<0.0001$ & $74.7 \pm 4.7$ & $<0.01$ \\
\hline Normal tissue & $4 / 30(13.3)$ & & $21.8 \pm 2.3$ & \\
\hline Non-tumorous & $19 / 50(38)$ & 0.018 & $36.23 \pm 3.6$ & $<0.01$ \\
\hline Normal tissue & $4 / 30(13.3)$ & & $21.8 \pm 2.3$ & \\
\hline \multicolumn{5}{|l|}{ Adenoma: } \\
\hline Tumorous & $7 / 14(50)$ & 0.04 & $57.73 \pm 5.6$ & $<0.01$ \\
\hline Non-tumorous & $2 / 14(14.2)$ & & $27.85 \pm 5.8$ & \\
\hline Tumorous & $7 / 14(50)$ & 0.009 & $57.73 \pm 5.6$ & $<0.01$ \\
\hline Normal tissue & $4 / 30(13.3)$ & & $21.8 \pm 2.3$ & \\
\hline Non-tumorous & $2 / 14(14.2)$ & 0.9 & $27.85 \pm 5.8$ & $>0.05$ \\
\hline Normal tissue & $4 / 30(13.3)$ & & $21.8 \pm 2.3$ & \\
\hline
\end{tabular}

$(\mathrm{P}>0.05)$ nor was higher in $\mathrm{CRC}$ than in adenoma $(\mathrm{P}>$ 0.05) (Table 4). This indicated that IL-8, unlike NF-кB, was not much associated with the transformation process of adenoma to CRC but, like NF- $\kappa \mathrm{B}$, was significantly associated with $S$. gallolyticus in CRC patients.

By means of Pearson's correlation, it was shown that the higher the level of S. gallolyticus IgG antibodies repre- sented by ELISA readings, the higher the expression of IL8 mRNA in the tumorous tissue sections of CRC patients; the correlation was significant and positive $(r=+0.32, \mathrm{P}<$ $0.05)$. Moreover, higher expression of IL- 8 mRNA in CRC was associated with more advanced stages of CRC $(\mathrm{P}<$ $0.05)$ but not with tumor grade $(P>0.05)$, in that CRC patients who showed high IL-8 mRNA expression (scores

Table 2: The expression of IL-8 mRNA in the tumorous and the non-tumorous tissue sections of CRC and adenoma patients as well as in normal tissue sections of control subjects.

\begin{tabular}{|c|c|c|c|c|}
\hline Tissue sections of IL-8 & $\begin{array}{l}\text { Median score of IL-8 } \\
\text { mRNA expression }\end{array}$ & $\begin{array}{l}\text { P value for Mann- } \\
\text { Whitney of ordinal } \\
\text { scores }\end{array}$ & $\begin{array}{c}\text { Mean percentage of IL-8 } \\
\text { mRNA positively stained } \\
\text { cells (\%) }\end{array}$ & $\begin{array}{c}\text { P value for positively } \\
\text { stained cells }\end{array}$ \\
\hline \multicolumn{5}{|l|}{ CRC: } \\
\hline Tumorous & Score 2 & 0.018 & $26.6 \pm 2.05$ & $<0.01$ \\
\hline Non-tumorous & Score 1 & & $9.28 \pm 1.3$ & \\
\hline Tumorous & Score 2 & 0.018 & $26.6 \pm 2.05$ & $<0.01$ \\
\hline Normal tissue & Score I & & $7.3 \pm 1.44$ & \\
\hline Non-tumorous & Score I & 0.56 & $9.28 \pm 1.3$ & $>0.05$ \\
\hline Normal tissue & Score I & & $7.3 \pm 1.44$ & \\
\hline \multicolumn{5}{|l|}{ Adenoma: } \\
\hline Tumorous & Score 2 & 0.018 & $23.71 \pm 1.86$ & $<0.01$ \\
\hline Non-tumorous & Score I & & $7.92 \pm 1.73$ & \\
\hline Tumorous & Score 2 & 0.018 & $23.71 \pm 1.86$ & $<0.01$ \\
\hline Normal tissue & Score I & & $7.3 \pm 1.44$ & \\
\hline Non-tumorous & Score I & 0.56 & $7.92 \pm 1.73$ & $>0.05$ \\
\hline Normal tissue & Score I & & $7.3 \pm 1.44$ & \\
\hline
\end{tabular}


Table 3: The expression of NF- $\kappa B$ mRNA in the tumorous and non-tumorous tissue sections of CRC versus adenoma patients and in CRC-Sg+ve versus CRC-Sg-ve groups.

\begin{tabular}{|c|c|c|c|c|}
\hline tissue sections & $\begin{array}{c}\text { Positive NF- } \kappa \text { B mRNA } \\
\text { expression } \\
\text { N (\%) }\end{array}$ & $\begin{array}{l}\chi^{2} \text { test } \\
P \text { value }\end{array}$ & $\begin{array}{l}\text { Mean \% of NFKB-positively } \\
\text { stained cells }\end{array}$ & $\begin{array}{l}\text { P value for positively stained } \\
\text { cells }\end{array}$ \\
\hline \multicolumn{5}{|c|}{ Tumorous tissue sections } \\
\hline \multicolumn{5}{|l|}{ Patients: } \\
\hline CRC & $36 / 50(72)$ & 0.042 & $74.7 \pm 4.7$ & $<0.01$ \\
\hline Adenoma & $7 / 14(50)$ & & $57.73 \pm 5.6$ & \\
\hline \multicolumn{5}{|c|}{ S. gallolyticus seropositivity } \\
\hline CRC-Sg+ve & $28 / 34(82)$ & 0.017 & $79.45 \pm 6.2$ & $<0.05$ \\
\hline CRC-Sg-ve & $8 / 16(50)$ & & $64.6 \pm 4.6$ & \\
\hline \multicolumn{5}{|c|}{ Non-tumorous tissue sections } \\
\hline \multicolumn{5}{|l|}{ Patients: } \\
\hline CRC & $19 / 50(38)$ & 0.09 & $36.23 \pm 3.6$ & $>0.05$ \\
\hline Adenoma & $2 / 14(14.2)$ & & $27.85 \pm 5.8$ & \\
\hline \multicolumn{5}{|c|}{ S. gallolyticus seropositivity } \\
\hline CRC-Sg+ve & $17 / 34(50)$ & 0.01 & $40.25 \pm 3.7$ & $<0.05$ \\
\hline CRC-Sg-ve & $2 / 16(12.5)$ & & $27.7 \pm 4.2$ & \\
\hline
\end{tabular}

2-3) were $3 / 8(37.5 \%)$ of stage $\mathrm{B}, 6 / 12$ (50\%) of stage C, and $24 / 30(80 \%)$ of stage D.

It is noteworthy to mention that the level of expression of tumor suppressor proteins p21, p27 and p53 which were examined previously on the same samples by Immunohistochemistry assay, [unpublished data] showed no asso- ciation with the seropositivity of $S$. gallolyticus in CRC patients.

ISH immunostaining of stromal cells

IL- 8 and NF- $\kappa$ B mRNA immunostaining of stromal cells rather than glandular mucosal cells (adeno cells) showed much lower levels than that of glandular mucosal cells in both tumorous and non-tumorous tissue sections of CRC

Table 4: The expression of IL-8 mRNA in the tumorous and non-tumorous tissue sections of CRC versus adenoma patients, and in CRC-Sg+ve versus CRC-Sg-ve groups.

\begin{tabular}{|c|c|c|c|c|}
\hline Tissue sections & $\begin{array}{l}\text { Median score of IL-8 } \\
\text { mRNA expression }\end{array}$ & $\begin{array}{l}\text { Mann-Whitney for } \\
\text { median scores } \\
(P \text { value })\end{array}$ & $\begin{array}{l}\text { Mean percentage of IL-8 } \\
\text { mRNA positively stained } \\
\text { cells } \\
(\%)\end{array}$ & $\begin{array}{l}\text { student } t \text { test for posi- } \\
\text { tively stained cells } \\
\text { (P value) }\end{array}$ \\
\hline \multicolumn{5}{|c|}{ Tumorous tissue sections } \\
\hline \multicolumn{5}{|l|}{ Patients: } \\
\hline CRC & Score 2 & 0.64 & $26.6 \pm 2.05$ & $>0.05$ \\
\hline Adenoma & Score 2 & & $23.7 \pm 1.86$ & \\
\hline \multicolumn{5}{|l|}{$\begin{array}{l}\text { S. gallolyticus } \\
\text { seropositivity: }\end{array}$} \\
\hline CRC-Sg+ve & Score 2 & 0.049 & $31.58 \pm 4.2$ & $<0.01$ \\
\hline CRC-Sg-ve & Score 1.5 & & $16 \pm 2.8$ & \\
\hline \multicolumn{5}{|c|}{ Non-tumorous tissue sections } \\
\hline \multicolumn{5}{|l|}{ Patients: } \\
\hline CRC & Score I & 0.56 & $9.28 \pm 1.3$ & $>0.05$ \\
\hline Adenoma & Score I & & $7.92 \pm 1.73$ & \\
\hline \multicolumn{5}{|l|}{$\begin{array}{l}\text { S. gallolyticus } \\
\text { seropositivity: }\end{array}$} \\
\hline CRC-Sg+ve & Score I & 0.56 & $10 \pm 2.9$ & $>0.05$ \\
\hline CRC-Sg-ve & Score I & & $7.56 \pm 1.7$ & \\
\hline
\end{tabular}


Table 5: The expression of NF- $K B$ and IL-8 mRNA in the stromal cells of the tumorous and non-tumorous tissue sections of CRC, adenoma, CRC-Sg+ve, and CRC-Sg-ve groups.

\begin{tabular}{lll}
\hline \multicolumn{1}{c}{ Tissue section } & \multicolumn{1}{c}{ Mean percentage of NF-KB mRNA expression } & \multicolumn{1}{c}{ Mean percentage of IL-8 mRNA expression } \\
$\%$ & \\
\hline Normal tissue & $21.8 \pm 2.3$ & $7.3 \pm 1.44$ \\
Tumorous CRC & $29.92 \pm 3.5$ & $11.55 \pm 1.07$ \\
Non-tumorous CRC & $23.2 \pm 2.8$ & $7.1 \pm 1.3$ \\
Tumorous adenoma & $24.15 \pm 2.7$ & $9.5 \pm 1.37$ \\
Non- tumorous adenoma & $23.06 \pm 1.6$ & $8.4 \pm 1.1$ \\
Tumorous CRC-Sg+ve & $30.41 \pm 2.3$ & $12.36 \pm 1.72$ \\
Ttumorous CRC-Sg-ve & $28.88 \pm 1.85$ & $9.84 \pm 1.25$ \\
Non-tumorous CRC-Sg+ve & $22.5 \pm 1.74$ & $7.2 \pm 1.21$ \\
Non-tumorous CRC-Sg-ve & $24.7 \pm 1.52$ & $6.8 \pm 0.62$ \\
\hline
\end{tabular}

and adenoma patients $(\mathrm{P}<0.05)$ (Table 5). The mRNA expression of IL-8 and NF-KB in stromal cells of CRC but not of adenoma patients was borderline higher in tumorous than both normal and non-tumorous tissue sections $(\mathrm{P}<0.05)$. However, there was no difference between non-tumorous and normal tissue sections in both CRC and adenoma patients $(\mathrm{P}>0.05)$ (Table 5). IL-8 mRNA and NF-kB mRNA expression in stromal cells of both tumorous and non-tumorous tissue sections was not different between CRC and adenoma patients and between CRC-Sg+ve and CRC-Sg-ve groups ( $\mathrm{P}>0.05)$ (Table 5). This indicated that mRNA expression of IL- 8 and NF- $\mathrm{\kappa B}$ in stromal cells, like glandular cells, showed association with CRC tumorous cells in comparison with non-tumorous and normal cells, however, unlike glandular cells, it showed no association with adenoma, the transformation process from adenoma to CRC, and no association with the seropositivity of $S$. gallolyticus IgG antibodies. This might be attributed to the fact that the involved CRC cases in this study were all of adenocarcinoma type.

\section{Discussion}

There are several microbial candidates, other than S. gallolyticus, for the association with CRC such as $S$. pasterianus, $S$. infantarius, $S$. salivarius, $H$. pylori, and some strains of $E$. coli $[2,3,7,9,17]$. However, except for $H$. pylori, the association of these bacteria has not been found as obvious as $S$. gallolyticus. Nevertheless, few studies have been conducted to investigate the serological aspect of $S$. bovis association with colorectal carcinoma and adenoma and no study has been conducted to investigate the serological association of $S$. gallolyticus with colorectal carcinoma and adenoma. The design of ELISA of the current study took into account the feasibility of measuring IgG antibodies towards $S$. gallolyticus and $B$. fragilis rather than IgM due to the expected long period of exposure to these bacteria, if any. It was previously shown that titers of IgM antibodies of $S$. bovis biotype I in CRC patients lack the consistency because the immune stimulation of CRC patients towards $S$. bovis has occurred over a long period of time [26].
In this study, the level of S. gallolyticus IgG antibodies was significantly higher in CRC and adenoma patients than in control and HV groups. Darjee and Gibb stated that patients with colonic cancer had higher median IgG antibody titers to $S$. bovis and E. faecalis preparations than control samples did [27]. Hence, the seroprevalence of IgG antibodies against $S$. gallolyticus subspecies $S$. gallolyticus, measured in the current study, showed the same behavior of the seroprevalence of IgG antibody against $S$. bovis NCTC 8133 (now is termed S. infantiarus). Moreover, the findings of the study of Devriese et al support our findings. They stated that $S$. gallolyticus is more likely to be involved in human infections which and associated with colonic cancer [8].

The association of seroprevalence of $S$. gallolyticus with CRC and colorectal adenoma in this study was specific because of the lack of similar seroprevalence association of the other studied bacteria, namely $B$. fragilis. $B$. fragilis was selected as one of the best candidate indicators for monitoring any history of breach of the mucosal barrier between large intestine and blood circulation by ulceration, tumor lesions, surgery, or necrosis. This draws our attention on how $S$. gallolyticus could be associated with $\mathrm{CRC}$ and adenoma. Is this association just a consequence of the tumor lesion or this bacterium might act as tumor initiator or promoter. Wanke and Bistrian revealed that local actions of cytokines or of chemical mediators able to promote vasodilatation and the enhancement of capillary permeability, may support bacterial entry at the tumor site, and increase bacterial adherence to various cells [28]. If this scenario was the explanation for the higher seroprevalence of $S$. gallolyticus in CRC and adenoma patients than in HV and control groups, we should have seen the same association for $B$. fragilis which is by far more dominant than S. gallolyticus in the bowel and of $100 \%$ incidence.

In addition, the association of S. gallolyticus with adenomatous polyps was another clue supporting the hypothesis 
of the association of $S$. gallolyticus with the transformation of colorectal mucosa from early adenomatous polyp stages to late CRC stages, taken into account that $90 \%$ of preinvasive neoplastic lesions of the colorectum are polyps or polyp precursors (aberrant crypt foci) [29]. The findings of this study were in agreement with other studies like Fagundes et al. who revealed that this bacterium among patients with septicemia and/or endocarditis is also related, in a significant way, to the presence of villous or tubulovillous adenomas in the large intestine [6]. Therefore, the serological investigation of $S$. gallolyticus in this study has shown 4 factors of reliability; the statistical significance of $S$. gallolyticus association with both adenomas and CRC, no similar association was seen by the indicator bacteria $B$. fragilis, relying on the measurement of IgG rather than IgM antibodies which reflects better the status of longstanding immune reaction against bacterial antigens, and the agreement of our results with other studies that based on isolating $S$. gallolyticus from blood at the bacteremic phase.

A colonoscopically proven control group was involved in order to obtain punch biopsies of normal mucosal tissues and also to calculate precisely the cutoff value of normal seropositivity of S. gallolyticus IgG antibodies. After applying this cutoff value on HV, CRC, and adenoma groups, it was apparent that such cutoff value can be used to screen the high risk groups of the population at sensitivity ranges from 68 to $78 \%$ for CRC and adenoma and at specificity of $83.33 \%$. Therefore, this assay can be used in designing an optimized kit for screening and/or detecting CRC or colorectal adenoma in the population by using simple, cheap and noninvasive procedure. Four seropositive subjects in HV group were examined by colonoscopy, 3 were normal and 1 revealed a medium-sized non-malignant hyperplasic polyp. The detected hyperplasic polyp might be an earlier event than adenoma for the association of $S$. gallolyticus with transformation of colonic mucosa. However, this needs a prospective study on an extensive population to determine on solid basis the association extent of benign polyps with $S$. gallolyticus.

The mRNA expression of NF- $\mathrm{kB}$ and IL-8 was significantly higher in tumor cells than in histologically normal adjacent cells in both CRC and adenoma patients and it was higher in CRC and adenoma than in control subjects. This provided evidence that NF- $\mathrm{KB}$ and IL- 8 might have a remarkable role in the transformation of colorectal adenoma and CRC. Several steps were dedicated to explore the nature of the association of NF- $\mathrm{KB}$ and IL-8 with colorectal adenoma and CRC in terms of relationship with $S$. gallolyticus. It was found that the expression of NF- $\mathrm{KB}$ mRNA in CRC-Sg+ve was significantly higher than in CRC-Sg-ve patients in both tumorous and non-tumorous tissue sections, and was higher in CRC than in adenoma patients in tumorous rather than non-tumorous tissue sections. These results were of high value by the virtue of many reasons. First, to the best of our knowledge, no previous reports were found to elucidate any possible relationship between NF-kB and S. gallolyticus in CRC and adenoma patients. Second, these findings pinpoint to a possibility that NF- $\kappa \mathrm{B}$ could be an essential factor for the carcinogenic effect of $S$. gallolyticus on colorectal mucosa. $\mathrm{NF}-\kappa \mathrm{B}$ is implicated in the transcription of many target genes that exert carcinogenic role and many studies revealed that the elevation of NF- $\kappa B$ activity was evident in a number of human cancers, including breast cancer [30], thyroid cancer [31], melanoma [32], and colon cancer $[33,34]$. Third, NF- $\mathrm{KB}$ mRNA expression was significantly higher in CRC than in adenoma patients indicating that NF- $\mathrm{KB}$ might play an important role in the transformation sequel from normal mucosa, to adenoma, and to malignant CRC stage, where NF- $\mathrm{BB}$ expression was highest.

The probable mechanism of carcinogenesis by $S$. gallolyticus via NF- $\mathrm{BB}$ is believed to be driven by the downstream mediators of NF- $\mathrm{kB}$. It was revealed that the synthesis of many cytokines and active compounds, which are transcriptionally dependent on NF- $\mathrm{KB}$, is in response to $S$. bovis and leads to the formation of COX-2, nitric oxide, and free radicals such as superoxide [35], peroxynitrites, and hydroxyl radicals [36], which all possess a highly potent mutagenicity. Furthermore, $S$. bovis was proved experimentally to induce ras oncogene [3] which requires the cell survival function of NF- $\mathrm{BB}$ to overcome death signal initiated in transformed cells [37]. Therefore, like $S$. bovis, it is possible that $S$. gallolyticus uses NF- $\mathrm{KB}$ as a survival tool against death signals along with its potentially mutagenic downstream mediators.

Regarding IL-8, its mRNA expression in tumorous rather than non-tumorous tissue sections of CRC-Sg+ve was significantly higher than in CRC-Sg-ve patients. Nevertheless, IL-8 mRNA expression in both tumorous and nontumorous tissue sections of CRC was not higher than that of adenoma patients. Pearson's correlation coefficient revealed that the level of $S$. gallolyticus IgG antibodies was positively correlated with IL-8 mRNA expression $(r+0.32$, $\mathrm{P}=0.022$ ) indicating that $S$. gallolyticus seroprevalence might be in parallel with IL-8 mRNA expression in CRC. Furthermore, higher expression of IL-8 mRNA in CRC was associated significantly with more advanced stages of CRC. These findings highlighted several interpretations. First, IL-8 is most probably associated with $S$. gallolyticus and correlated well with its seroprevalence level which might indicate a role to play for the association between this bacterium and CRC. Second, unlike NF- $\kappa \mathrm{B}, \mathrm{IL}-8$ association with $S$. gallolyticus was restricted to tumorous rather than non-tumorous cells which might indicate a 
propagating rather than promoting oncogenic role of $S$. gallolyticus via IL-8. Third, in supporting the earlier analysis, the level of IL- 8 mRNA was not significantly different between adenoma and CRC patients in both tumorous and non-tumorous tissue sections while it was clearly associated with disease progression and CRC staging.

The pattern of IL- 8 mRNA expression led us to propose that IL-8 is more likely a propagating factor for CRC and lacks the promoting role in the transformation process from colorectal adenoma to CRC. This might be attributed to the angiogenic role of IL- 8 by which new blood vessels are formed to meet the increasing demands of cancer growth. These findings are supported by Ellmerich et al. who revealed that small amounts of IL- 8 were detected in the mucosa of control rats, while the level of IL- 8 was $\sim 4$ to 3 folds higher in the mucosa of rats receiving $S$. bovis and $S$. bovis wall extracted antigen fraction, respectively [38]. On the other hand, because IL-8 transcription was shown to be dependent on the cooperative action of two transcription factors, NF- $\mathrm{B}$ and AP-1 [39], hence, both NF- $\kappa \mathrm{B}$ and IL- 8 might play an integrated role leading to a series of steps to escape from death signals, release of potentially mutagenic products, and preparing the needed blood supply to the cancerous cells. Regarding the ISH staining of stromal cells, they were found to express mRNA of NF- $\kappa$ B and IL- 8 much lower than that of glandular mucosal cells. Although mRNA expression of NF- $\kappa B$ and IL-8 was not associated in stromal cells with $S$. gallolyticus IgG antibodies, the NF- $\mathrm{B}$ and IL-8 expression was higher in tumorous CRC than in tumorous adenoma sections. Accordingly, glandular rather than stromal cells have shown to be mainly responsible for the expression of NF- $\kappa \mathrm{B}$ and IL- 8 as well as glandular rather than stromal cells seem to play the major role in the association of $S$. gallolyticus with colonic mucosa.

\section{Conclusion}

Taken together, S. gallolyticus subspecies gallolyticus was found serologically associated with both CRC and colorectal adenoma. The seroprevalence of S. gallolyticus IgG antibodies appeared as a reliable indication for the association of this bacterium with CRC and adenoma. Moreover, S. gallolyticus has shown a specific association with CRC and colorectal adenoma when compared with the more dominant intestinal bacteria, B. fragilis. This provided evidence for a possible important role of $S$. gallolyticus in the carcinogenesis of CRC from pre-malignant polyps. In addition, the seroprevalence of $S$. gallolyticus has shown to be a good candidate for formulating a screening assay for the early detection of both adenoma and CRC cases in high risk groups. Regarding the possible underlying mechanisms of such association, NF- $\kappa \mathrm{B}$ and IL-8 rather than other transformation factors p21, p27 and p53 [unpublished data] might highly act as important mediators for the S. gallolyticus-associated carcinogenesis of adenoma to carcinoma. Moreover, it was concluded that NF- $\kappa \mathrm{B}$ exerts most probably a promoting carcinogenic effect and IL-8 exerts mainly a propagating effect on colorectal mucosal cells. We recommend conducting further studies to reveal the in vitro effect of $S$. gallolyticus on colorectal mucosal cells in relation to factors such as COX2, Ras, apoptosis markers, PGE2, TNF-alpha, IL-1, and IL6 . We also recommend conducting a prospective screening study on the high risk groups of the population using the formulated ELISA kit to measure the seropositivity of S. gallolyticus IgG antibodies and assess the reliability of such screening assay when conducted on a large scale sample of the population.

\section{Competing interests}

The authors declare that they have no competing interests.

\section{Authors' contributions}

AS carried out the sampling, taking the medical history, patients examination, and supervision on the immunological tests. AS, RR, and LK carried out the bacteriological cultures, cell wall extraction, and LPS extraction. T and LK supervised the immunological testing and edited the outline of the manuscript. AS, RR and F carried out the pathological and histological examination, the classification of the biopsies, and ISH. AS, RR, and T carried out the statistical design, statistical analysis, and the design of the cut off value. All authors read and approved the final manuscript.

\section{Acknowledgements}

This study was designed and performed in Malaysia; however, this study was partially carried out in the laboratories of the department of Bacteriology and hospital hygiene, CHU Hospital Trousseau, Tours, France. Scientific assistance was kindly supplied by the chief of department, Prof. Roland Quentin and all other staff members.

\section{References}

I. Crawford JM, Kumar V: The oral cavity and the gastrointestinal tract. In Basic Pathology Seven edition. Edited by: Kumar V, Cotran RS, Robbins SL. Philadelphia and London: Saunders and Elsevier Science; 2003:543-590.

2. Newman JV, Kosaka T, Sheppard BJ, Fox JG, Schauer DB: Bacterial infection promotes colon tumorigenesis in Apc (Min/+) mice. J Infect Dis 200I, I 84:227-30.

3. Jordane B, Nguyen IS, Pini A, Gossé F, Richert S, Thiersé D, Van Dorsselaer A, Leize-Wagner E, Raul F, Klein JP, Schöller-Guinard M: Carcinogenic properties of proteins with pro-inflammatory activity from Streptococcus infantarius (formerly S. bovis). Carcinogenesis 2004, 25:|477-84.

4. Leport C, Bure A, Leport J, Vilde JL: Incidence of colonic lesions in Streptococcus bovis and enterococcal endocarditis. The Lancet 1997, I:748.

5. Zarkin BA, Lillimoe KD, Cameron JL, Efforon PN, Magnuson TH, Pitt HA: The triad of Streptococcus bovis bacteremia, colonic pathology, and liver disease. Ann Surg 1990, 2 I I:786-92.

6. Fagundes J], Noujain HM, Coy CSR, Ayrizono MLS, Góes JRN, Martinuzzo WRG, Costa AM, Medeiros RR, Leonardi LS: Association between bacterial endocarditis and neoplasia in relation to 4 cases. Rev Bras Coloproctol 2000, 20:95-9. 
7. Ruoff KL, Miller SI, Garner CV, Ferraro MJ, Calderwood SB: Bacteremia with Streptococcus bovis and Streptococcus salivarius: clinical correlates of more accurate identification of isolates. J Clin Microbiol 1989, 27:305-308.

8. Devriese LA, Vandamme P, Pot B, Vanrobaeys M, Kersters P, Haesebrouck F: Differentiation between Streptococcus gallolyticus strains of human clinical and veterinary origins and Streptococcus bovis strains from the intestinal tracts of ruminants. J Clin Microbiol 1998, 36:3520-3523.

9. Schlegel L, Francine G, Elisabeth A, Patrick AD: Reappraisal of the taxonomy of the Streptococcusbovis/Streptococcus equinus complex and related species: description of Streptococcus gallolyticus subsp. gallolyticus subsp. nov., S. gallolyticus subsp. macedonicus subsp. nov. and $S$. gallolyticus subsp. pasteurianus subsp. nov. Int J Syst Evol Microbiol 2003, 53:63 I-645.

10. Brook I: Clinical review: bacteremia caused by anaerobic bacteria in children. Crit Care 2002, 6:205-2II.

I I. Linko-Kettunen L, Arstila P, Jalkanen M, Jousimies-Somer H, Lassila O, Lehtonen OP, Weintraub A, Viljanen MK: Monoclonal antibodies to Bacteroides fragilis lipopolysaccharide. J Clin Microbiol I984, 20:519-24.

12. GenWay Biotech, Inc [http://www.genwaybio.com/ product info.php?products id $=73049]$

13. Westrin KM, Stierna P, Weintraub A, Palmgren AC, Nord CE: Serum antibody response to Bacteroides fragilis in experimental sinusitis. Infection 1991, 19:435-9.

14. Delahooke DM, Barclay GR, Poxton IR: Tumor necrosis factor induction by an aqueous phenol-extracted lipopolysaccharide complex from Bacteroides species. Infect Immun 1995, 63:840-6.

15. Abdulamir AS, Hafidh RR, Mahdi LK, Aljeboori T, Abubakar F, Abbas KA: The Interplay between p53 and p2I Tumor Suppressor Proteins in the Transformation of Colorectal Adenoma to Carcinoma. Am J Immunol 2008, 4:14-22.

16. Nichols TC, Fischer TH, Deliargyris EN, Baldwin AS Jr: Role of nuclear factor-kappa B (NF-kappa B) in inflammation, periodontitis, and atherogenesis. Ann Periodontol 200I, 6:20-29.

17. Bartchewsky W Jr, Martini MR, Masiero M, Squassoni AC, Alvarez MC, Ladeira MS, Salvatore D, Trevisan M, Pedrazzoli J Jr, Ribeiro ML: Effect of Helicobacter pylori infection on IL-8, IL-I beta and COX-2 expression in patients with chronic gastritis and gastric cancer. Scand J Gastroenterol 2009, 44:|53-|6|.

18. Srinivasan M, Sedmak D, Jewell S: Effect of fixatives and tissue processing on the content and integrity of nucleic acids. Am J Pathol 2002, 161: 1961-71.

19. Lisowski AR, English ML, Opsahl AC, Bunch RT, Blomme EA: Effect of the storage period of paraffin sections on the detection of mRNAs by in situ hybridization. I Histochem Cytochem 200I, 49:927-8.

20. Vanbrobaeys M, Haesebrouck F, Ducatelle R, De Herdt P: Identifcation of virulence associated markers in the cell wall of pigeon Streptococcus gallolyticus strains. Vet Microbiol 2000, 73:319-325.

21. Weintraub A, Larsson BE, Lindberg AA: Chemical and immunochemical analyses of Bacteroides fragilis lipopolysacharides. Infect Immune 1985, 49: I 97-20I.

22. Schuurs A, Weeman V: Enzyme innunoassay. Clin Chem Acta 1977, $3 \mathrm{I}: \mathrm{I}-40$.

23. Al-Murrani WK, Al-Shummari A, Al-Obaidi A, Mustafa AM: New approach for the calculation of the cutoff value(value) in immunological and diagnostic tests. Iraqi J Microbiol 2000, 12:1-9.

24. Kadhim H, Aljeboori T, Tawfik M: Possible role of cell cycle regulatory proteins and nuclear factor $-\kappa B$ on the pathogenesis of transitional cell carcinoma of the bladder. In PhD Thesis AlNahrain University, Microbiology Department; 2004.

25. Konstantinovsky S, Søren N, Mogens V: Angiogenic molecule expression is downregulated in effusions from breast cancer patients. Breast Cancer Res Treat 2005, 10:322-28.

26. Panwalker AP: Unusual infections associated with colonic cancer. Rev Infect Dis 1991, 10:347.

27. Darjee R, Gibb AP: Serological investigation into the association between Streptococcus bovis and colonic cancer. J Clin Pathol 1993, 46: I II6-9.

28. Wanke CA, Bistrian B: Recombinant human tumor necrosis factor and recombinant murine interleukin-I alter the bind- ing of Escherichia coli to intestine, mucine glycoprotein, and the HT29-CI intestinal cell line. Nutrition 1997, I 3:959-964.

29. Grizzle WE, Shibata D, Manne U: Molecular and Histopathologic Changes in the Development of Colorectal Neoplasia. In Molecular Pathology of Early Cancer Ist edition. Edited by: Srivastava S, Henson DE, Gazdar A. Washington DC: IOS Press; 1999:135-170.

30. Sovak MA, Bellas RE, Kim DW, Zanieski GJ, Rogers AE, Traish AM, Sonenshein GE: Aberrant nuclear factor-_B/Rel expression and the pathogenesis of breast cancer. J Clin Invest 1997, 100:2952-2960.

31. Gilmore TD, Morin PJ: The I-KB protein: members of a multifunctional family. Trends Gene 1993, 9:427-433.

32. Devalaraja MN, Wang DZ, Ballard DW, Richmond A: Elevated constitutive $I-\kappa B$ kinase activity and $I \kappa B$ phosphorylation in Hs294T melanoma cells lead to increased basal MGSA/GROtranscription. Cancer Res 1999, 59:1372-1377.

33. Dejardin E, Deregowski V, Chapelier M, Jacobs N, Gielen J, Merville MP, Bours V: Regulation of NF- $\kappa$ B activity by I $\kappa$ B-related proteins in adenocarcinoma cells. Oncogene 1999, 18:2567-2577.

34. Wang Wei, He-Sheng L, Bao-Ping Yu: Expression of NF-kB and human telomerase reverse transcriptase in gastric cancer and precancerous lesions. World J Gastroenterol 2004, 10: I77- I8I.

35. Janeway CA, Travers P: Immunobiology: the Immune System in Health and Disease Garland Publishing Inc: New Yorkand London; 1997.

36. Ohshima $H$, Bartsch $H$ : Chronic infections and inflammatory processes as cancer risk factors: possible role of nitric oxide in carcinogenesis. Mutat Res 1994, 305:253-264.

37. Chen F, Castranova V, Shi $X$, Demers LM: New insights into the Role of Nuclear Factor- $\kappa B$, a Ubiquitous Transcription Factor in the Initiation of Diseases. Clin Chem 1999, 45:7-17.

38. Ellmerich S, Schöller M, Duranton B, Gossé F, Galluser M, Klein JP, Raul F: Promotion of intestinal carcinogenesis by Streptococcus bovis. Carcinogenesis 2000, 21:753-756.

39. Mukaida N: Interleukin-8: an expanding universe beyond neutrophil chemotaxis and activation. Int J Hematol 2000, 72:391-398.

\section{Pre-publication history}

The pre-publication history for this paper can be accessed here:

http://www.biomedcentral.com/1471-2407/9/403/pre pub
Publish with Bio Med Central and every scientist can read your work free of charge

"BioMed Central will be the most significant development for disseminating the results of biomedical research in our lifetime. "

Sir Paul Nurse, Cancer Research UK

Your research papers will be:

- available free of charge to the entire biomedical community

- peer reviewed and published immediately upon acceptance

- cited in PubMed and archived on PubMed Central

- yours - you keep the copyright
BioMedcentral 